Meta

Journal des traducteurs

Translators' Journal

\title{
Index des mots et sujets traités dans le volume 18
}

Volume 18, numéro 4, décembre 1973

URI : https://id.erudit.org/iderudit/003212ar

DOI : https://doi.org/10.7202/003212ar

Aller au sommaire du numéro

Éditeur(s)

Les Presses de l'Université de Montréal

ISSN

0026-0452 (imprimé)

1492-1421 (numérique)

Découvrir la revue

Citer ce document

(1973). Index des mots et sujets traités dans le volume 18. Meta, 18(4), 396-404.

https://doi.org/10.7202/003212ar d'utilisation que vous pouvez consulter en ligne.

https://apropos.erudit.org/fr/usagers/politique-dutilisation/ 


\section{INDEX DES MOTS \\ ET SUJETS TRAITES DANS LE VOLUME 18}

Abeille, 4, p. 372.

Aboiteau, 1-2, p. 182.

Absorption, 3, p.336.

Acceptions mélioratives, 1-2, p. 193.

Acceptions péjoratives, 1-2, p. 189.

Accident en cours de route, 4, p. 379.

Accommodement, 4, p. 379.

Accréditer, 4, p. 379.

Acier (glossaire sur), 1-2, p. 251.

Acre, 1-2, p. 185.

Adaptation à l'autre, 1-2, p. 212.

Adjectivation du substantif, 1-2, p. 204.

Adjectivité aiguë, 1-2, p. 246.

Adresse, 1-2, p. 92.

Adsorption, 3, p. 336.

Affectif et intellectuel, 1-2, p. 172.

Affectivité, 3, p. 315.

Affinage des matériaux linguistiques, 1-2, p. 98.

Age des neutrons, 1-2, p. 241.

Agrégatif, 1-2, p. 258

Agriflamme, 1-2, p. 259.

Aire sémantique, 1-2, p. 177.

Allée, 1-2, p. 178.

"Altérations ", 4, p. 372.

Amendement, 4, p. 379.

Amener, 1-2, p. 175

Amplificateur d'audiofréquence, 4, p. 366.

Analogie, 1-2, p. 209.

Analyse automatique, 1-2, p. 98.

Analyse componentielle, 1-2, p. 147

Analyse distributionnelle, 1-2, p. 49 .

Analyse du groupe verbal, 1-2, p. 282

Analyse du lexique, 1-2, p. 19.

Analyse en sèmes, 1-2, p. 148 .

Analyse formelle, 1-2, p. 49

Analyse (homographies), 1-2, p. 93.

Analyse morphologique de l'anglais, 1-2, p. 279.

Analyse phonologique, 1-2, p. 147

Analyse (polysémies), 1-2, p. 93 .

Analyse syntaxique de l'anglais, 1-2, p. 281.

Ancien écossais, 1-2, p. 97.

Anglais de base, 1-2, p. 259.

Anglicismes, $1-2$, p. 231 .

Anglicismes honteux, 1-2, p. 229

Année financière, 4, p. 383 .

"Année físcale », 4, p. 383.

Anse, 1-2, p. 178.

Anti, 1-2, p. 227.

Anticulture, 1-2, p. 228.

Antipollution. 1-2, p. 228.

Antipsychiatrie, 1-2, p. 228.

Anti-vacance, $1-2$, p. 228 .

Antonymie, 1-2, p. 209.

Appartement, 1-2, p. 230

Applications de l'informatique à la lexicologie, 1-2, p. 87

Aquatique, 4, p. 370.

Aquiculture, 3 , p. 335 .
Archives, 1-2, p. 96

Archives mécanographiques, 1-2, p. 90, 91.

Argot, 4, p. 362.

Article de dictionnaire, 1-2, p. 130

Articulation du lexique, 1-2, p. 10.

Assiette anglaise, 1-2, p. 183.

Association des traducteurs et interprètes de l'Ontario (A.T.I.O.), 4, p. 394

Association des traducteurs littéraires de France, 4, p. 393.

Association internationale des interprètes de conférence (A.I.I.C.), 3, p. 349.

Assurances sociales, 3 , p. 340 .

Atome chaud, 1-2, p. 241.

Atome (qualification de 1'), 1-2, p. 244.

Atomicien, 1-2, p. 246.

Atomique, 1-2, p. 245.

Attache, 4, p. 383.

Attraction paronymique, $1-2$, p. 228

Audio-médial, 3, p. 312.

Audio-visuel, 3, p. 309.

Autarcie linguistique, 1-2, p. 225

Auteur/écrivain, 1-2, p. 174.

Automatisation de la recherche lexicologique, $1-2$, p. 103.

Automatisation et lexicologie, 1-2, p. 7.

Autonomie fonctionnelle du sémème, 1-2, p. 209.

Autonyme, 1-2, p. 54, 55.

Autopsie, 1-2, p. 197.

Auxiliaire copulatif, 1-2, p. 45.

"Avantages marginaux ", 1-2, p. 273.

Avenir de la TA, 1-2, p. 287.

Avertissement formel, 4, p. 379.

Ballet \& aquatique », 4, p. 371.

Ballet nautique, 4 , p. 371 .

Ballon panier, 1-2, p. 258

Bande, 1-2, p. 92.

Banque, 1-2, p. 176.

Banque de données, 1-2, p. 96, 101.

Banque de mots, 1-2, p. 131.

Barachois, 1-2, p. 182.

Barbiturique, 4 , p. 359

Barème de correction, 4, p. 393

Barre de torsion, 1-2, p. 259.

* Basse » fréquence, 4, p. 366.

Bâtiment, 1-2, p. 176

Bébé-éprouvette, 1-2, p. 259

Behaviorisme linguistique, 1-2, p. 157.

Belligérant, 1-2, p. 172

Bénéfice, 4, p. 379.

Besoins du milieu en terminologie, 1-2, p. 270.

Biberon, 1-2, p. 178.

Bibliographie de l'informatique linguistique, 1-2, p. 134.

Bibliographies raisonnées, 1-2, p. 273.

Bibliothèque électronique, 1-2, p. 111.

Bic, 4, p. 360.

Biennale de Dakar, 3 , p. 347.

Bilinguisme, 1-2, p. 212. 
Billard, 1-2, p. 176.

Bimestriel, 4, p. 379.

Biomasse, 3, p. 335.

Bleutière, 1-2, p. 185

Bobine, 1-2, p. 274.

Bonhomme d'Ampère, 1-2, p. 192.

Border, 1-2, p. 177.

Bordure de trottoir, 1-2, p. 177.

Bottin, 1-2, p. 58 .

Boucher, Emile, 3, p. 346

Bourgot, 1-2, p. 182.

Bouton, 1-2, p. 177.

Bras de lecture, 4, p. 366.

Brûler, 1-2, p. 204.

Brûlot, 1-2, p. 185.

Brunette, 1-2, p. 170.

Bulletins de terminologie, 4, p. 394.

Bureau (termes de), 4, p. 381 .

Cabas, 1-2, p. 178.

Cadres, 3, p. 329, 330.

Calque morphologique, 1-2, p. 230.

Calque sémantique, $1-2$, p. 230

Canadianismes, 1-2, p. $179,183,258 ; 4$, p. 378.

Capital de roulement d'une banque de terminologie, 1-2, p. 271 .

Capteur, 4, p. 366.

Caractérisation, 1-2, p. 177

Carapace, 1-2, p. 177.

Cartes mécanographiques, 1-2, p. 90.

Cartouche, 4, p. 366.

Catégorisation grammaticale, 1-2, p. 210.

Causer, 1-2, p. 213.

Cellule lectrice, 4 , p. 366.

Cellule phonolectrice, 4 , p. 366.

Centres universitaires de calcul, 1-2, p. 101.

C.E.S., 4, p. 369.

Chaîne, 4, p. 373

Chambre à coucher, 1-2, p. 176

Champ sémasiologique, 1-2, p. 75.

Charcuterie, 1-2, p. 175 .

Charge alaire, 1-2, p. 259

Charger, 4, p. 360.

Cheminée, 1-2, p. 176.

Chèque-repas, 1-2, p. 260

Cheval, 1-2, p. 211.

Chnouf, 4, p. 362 .

Choc, 1-2, p. 198.

Chronologie, 1-2, p. 68.

Circonstant, 1-2, p. 282

Cimetière fertile, 1-2, p. 241.

Citations et dictionnaire, 1-2, p. 75.

Civilisation du langage, 1-2, p. 234.

Classe, 1-2, p. 176

Classement des significations, 1-2, p. 75 .

Classement onomasiologique, 1-2, p. 82.

Classement sémasiologique, 1-2, p. 82.

Clause de conscience, 4, p. 379.

Clichés radiologiques, 1-2, p. 264.

"Clip-cave », 1-2, p. 192.

Clip-fémorale, 1-2, p. 192.

Clip-jugulaire, 1-2, p. 192.

Coin de feu, 1-2, p. 176.

Col claudine, 4, p. 372.

Collège, 4, p. 368 .

Collège classique, 4 , p. 369 .

Collège communal, 4, p. 369.
Collège d'enseignement général et professionnel (C.E.G.E.P.), 4, p. 370 .

Collège d'enseignement secondaire (C.E.S.), 4 , p. 369.

Col officier, 4 , p. 373.

Combinatoire syntaxique, $1-2$, p. 210

Communication (langage et), 1-2, p. 22.

Commutation de la forme signifiante, 1-2, p. 209.

Comparaison, 3 , p. 324

Compendieusement, 4, p. 360.

Compétence lexicale, $1-2$, p. 16 .

Compétence linguistique, $1-2$, p. 150

Compétence syntaxique, 1-2, p. 16.

Comportement dénominatif, 1-2, p. 25

Composante sémantique du terme, 3, p. 318.

Composition, 1-2, p. 207, 218.

Compositionalité (principe de), 1-2, p. 146.

Composition syntagmatique, 1-2, p. 248.

Composition syntagmatique asyndétique, 1-2, p. 249.

Composition syntagmatique avec joncteur, $1-2$, p. 249.

Composition syntagmatique épithétique, 1-2, p. 249.

Comptabilisation du système de la Banque de terminologie, $1-2$, p. 275

Concentration, 1-2, p. 171.

Concordance, 1-2, p. 89, 94, 107.

Concordance de formes, 1-2, p. 107.

Concordance différentielle, 1-2, p. 95.

Concret, 3, p. 315, 316

Conférence, 3, p. 332 .

Conférence internationale permanente des directeurs d'instituts universitaires pour la formation de traducteurs et d'interprètes (C.I.U.T.I.), 3, p. 347.

Confrontation, 3, p. 311 .

Congés payés, 4 , p. 380.

Connaissance et compréhension, 3, p. 310.

Connotation, 1-2, p. 157.

Conscience, 1-2, p. 176.

Conseil des traducteurs et interprètes du Canada (C.T.I.C.), 3, p. 348.

Conseil international de la langue française (C.I.L.F.), 3, p. 344.

Construction mécanique, 1-2, p. 264.

Consultation d'un lexique automatique, 1-2, p. 115.

Consultation en différé, 1-2, p. 275.

Consultation en accès direct, 1-2, p. 275.

Consultation téléphonique, $1-2$, p. 275.

Contenu sémantique, 1-2, p. 153.

Contexte, 1-2, p. 112.

Contexte en terminologie, 1-2, p. 269.

Contextes fixes, 1-2, p. 98 .

Contextes variables, $1-2$, p. 98 .

Contextualisation, 1-2, p. 111 .

Contre, 1-2, p. 227.

Contre-culture, 1-2, p. 228.

Contre-information, 1-2, p. 228.

Contretout, 1-2, p. 228 .

Conventions collectives, 4, p. 291.

Conventions typographiques, 4 , p. 109.

Convertir, 4, p. 365.

Co-occurrences, 1-2, p. 98.

Co-occurrents contextuels, 1-2, p. 98 .

Coordination des projets, 1-2, p. 102. 
Copistes (fautes de), 1-2, p. 67.

Coquetier, 1-2, p. 178.

Coquille, 1-2, p. 177.

Cordon de soudure, 1-2, p. 263.

Corps gras, 3, p. 342.

Corps gris, 1-2, p. 241

Corps noir, 1-2, p. 241.

Corpus, 1-2, p. 129.

Corrections des données enregistrées, 1-2, p. 110.

Corrections des épreuves, 1-2, p. 108.

Correspondance de traits, 1-2, p. 282.

Corrosion préférentielle, 1-2, p. 264.

Coup de filet, 1-2, p. 266.

Courant électrique, 4, p. 365 .

Cours, 3, p. 332 .

Court-circuitage par d'adjectif, 1-2, p. 264.

Couture anglaise, 4 , p. 372.

Couture (termes de), 4 , p. 372

Crayon-feutre, 1-2, p. 260.

Création néologique, 1-2, p. 206.

Création lexicale, 1-2, p. 218.

Crêperie, 1-2, p. 260.

Cristallographie, 1-2, p. 263.

Croquet, 4, p. 372 .

Cryo, 1-2, p. 196

Dé-, 1-2, p. 227

Décarburation, 1-2, p. 248.

Décélération, 1-2, p. 228.

Découpage de la réalité, 1-2, p. 251.

Décrément logarithmique moyen de l'énergie, $1-2$, p. 240.

Dédoublement, 3 , p. 325.

Définition, 1-2, p. 149

Définition de la lexicologie, 1-2, p. 9.

Définition double, $1-2$, p. 66 .

Définition et contexte, 1-2, p. 270

Définition et références terminologiques, 1-2, p. 161.

Définition fausse, 1-2, p. 66.

Définition (télévision), 1-2, p. 163.

Déformation de trempe, 1-2, p. 248.

Degré, 3, p. 318.

Délai de réponse, 1-2, p. 275.

Délectuaire, 4, p. 360 .

Délimitation des aires sémantiques, 1-2, p. 174.

Demiard, 1-2, p. 185.

Demi-produit, 1-2, p. 247.

Démologie, 1-2, p. 104

Démon, 1-2, p. 192.

Démon de Maxwell, 1-2, p. 192.

Dénomination, $1-2$, p. 54

Densité, $1-2$, p. 170

Déploiement d'un parachute, 1-2, p. 270.

Dépôt cumulatif des données, $1-2$, p. 100.

Dépôt de la convention, 4, p. 381

Dépouillement lexical, 1-2, p. 107, 114

Dépouillement (résultats), 1-2, p. 108.

Dépréciatif, 3, p. 319

Dérivation, $1-2$, p. 248

Dérivation par affixe, 1-2, p. 207.

Dérivation syntagmatique, $1-2$, p. 241.

Dérivés (statut des), 1-2, p. 82 .

Désacralisation, $1-2$, p. 228.

Description du lexique, $1-2$, p. 9.

Désescalade, 1-2, p. 228.

Désétatisation, 1-2, p. 228
Désignant, 1-2, p. 239.

Désignation, 1-2, p. 239.

Désignation et signification, 1-2, p. 150.

Dessous-de-table, 1-2, p. 260.

Détermination automatique des contextes, 1-2, p. 98.

Diable, 1-2, p. 192

Diachronie, 1-2, p. 213.

Dialectologie, 1-2, p. 71, 104.

Dialogue avec l'ordinateur, 1-2, p. 99.

Diathè̀que, $1-2$, p. 218 .

Dicautom, 1-2, p. 262.

Dictionnaire, 1-2, p. 201.

Dictionnaire alphabétique, 1-2, p. 61.

Dictionnaire analogique, 3, p. 311 .

Dictionnaire bilingue, 1-2, p. 179.

Dictionnaire chronologique, 1-2, p. 94.

Dictionnaire conceptuel, 1-2, p. 61.

Dictionnaire contextuel, 1-2, p. 216.

Dictionnaire de fréquences, $1-2$, p. 95 .

Dictionnaire diachronique, 1-2, p. 61.

Dictionnaire diachronique (sources), 1-2, p. 63.

Dictionnaire dynamique, 1-2, p. 100.

Dictionnaire et norme, 1-2, p. 220 .

Dictionnaire génétique, $1-2$, p. 61 .

Dictionnaire-machine, $1-2$, p. 92

Dictionnaire ouvert, 1-2, p. 100.

Dictionnaire (rôle du), 1-2, p. 51 .

Dieu, 1-2, p. 193.

Diffusion, 1-2, p. 271.

Diplômés en traduction, 3 , p. 350 .

Direction, 3, p. 329, 330.

Discours, 1-2, p. 201

Disques, 1-2, p. 92.

Documentation, $1-2$, p. 89,97 .

Documentation automatique, 1-2, p. 104.

Documentation automatique juridique, 1-2, p. 104.

Documentation massive, 1-2, p. 97.

Domaines d'application, 1-2, p. 273.

Double articulation, $1-2$, p. 56 .

Doul, doulos, 4, p. 362 .

Droit fil, 4 , p. 373.

Duplicater, 1-2, p. 236.

Dur (physique nucléaire), 1-2, p. 241.

Durée de chauffage, $1-2$, p. 247

Durée de mise en température, 1-2, p. 247.

Ecaille, 1-2, p. 177

Ecart de registre, 1-2, p. 172

Ecart de sens, 1-2, p. 172

Echantillon, 1-2, p. 136.

Echantillonnage, 1-2, p. 259

Ecole néobloomfieldienne, 1-2, p. 20.

Economie, 1-2, p. 264.

Economie par l'évidence, 1-2, p. 175.

Ecrivain, 1-2, p. 174.

Editions standardisées, 1-2, p. 68.

Education des adultes, 4, p. 380.

Education permanente, 4, p. 379.

Elaboration des résultats du dépouillement, 1-2, p. 108

Elan d'Amérique, 1-2, p. 183.

Electricité, 4, p. 390.

Electroacoustique, 4, p. 365 .

Electuaire, 4, p. 360 .

Eléments d'entrée, 1-2, p. 92.

Emmener, 1-2, p. 175. 
Employé, 4, p. 379.

Employé de bureau, 4, p. 380.

Emprunt, 1-2, p. $56,57,229,231$; 4, p. 358.

Emprunt lexical, 1-2, p. 56

Enseignement du français, 1-2, p. 233.

Ensemble, 1-2, p. 198.

"Ensemble de chambre ", 1-2, p. 175.

Entente formelle, 4, p. 379.

Entente officieuse, 4, p. 379.

Enterrement, 1-2, p. 172.

Entrée, 1-2, p. 107.

Entrée de dictionnaire, 1-2, p. 232.

Entrée des données, 1-2, p. 92.

Entretenir (s'), 1-2, p. 214.

Environnement, 3, p. 335.

Epreuve hydraulique, 1-2, p. 263.

Eprouvette, 1-2, p. 270.

Erothèque, 1-2, p. 228.

Espéranto, 3, p. $338 ; 4$, p. 356.

Esprit de finesse, 1-2, p. 158

Esprit de géométrie, 1-2, p. 158

Etage, 1.2, p. 174

Etat fondamental, 1-2, p. 240

Etat normal, 1-2, p. 240.

Etre noir aux neutrons, 1-2, p. 241.

Etymologie fausse ou double, 1-2, p. 65.

Etymon, 1-2, p. 207.

Etymon et définition, 1-2, p. 72

Evolution, 1-2, p. 226

Examen professionnel, 4, p. 393.

Exclusivité syndicale, 4, p. 380 .

Exemples d'auteur, 1-2, p. 75.

Exemples forgés, 1-2, p. 75

Exode des cerveaux, 1-2, p. 230.

Exploitation, 1-2, p. 190.

Exploitation (banque de terminologie), 1-2, p. 274.

Expressivité, 3, p. 315.

Extraposition, 1-2, p. 36

Facteur de désavantage, 1-2, p. 240.

Facteur de diffusion du néologisme, 4, p. 358.

Facteur de flux neutronique, 1-2, p. 240.

Faits lexicaux (nature des), 1-2, p. 19.

Famille de mots, 1-2, p. 62, 82 .

Fanes de carotte, 1-2, p. 178

Fautes de copistes, 1-2, p. 67.

Fauteuil, 1-2, p. 210

Fédération internationale des traducteurs (F.I.T.) , 4, p. 394.

Fémelitude, 1-2 p. 228

Ferme, 1-2, p. 47 .

Fermeture à glissière, 4, p. 372 .

Fermeture-éclair, 4, p. 372.

Ferrer, 1-2, p. 185.

Fiabilité, 1-2, p. 199

Fiable, 1-2, p. 194.

Fiche-contexte, 1-2, p. 107

Fiche et banque de terminologie, 1-2, p. 272.

Fiche terminologique, 1-2, p. 178, 272 .

Figuré et propre, 1-2, p. 172.

Folklore, 4, p. 357.

Fonction dénominative, 1-2, p. 22.

Font, 1-2, p. 109.

Formation au commandement, 3, p. 331.

Formation de cadres, 3, p. 329.

Formation de sujet, 1-2, p. 44.

Formation diminutive, 1-2, p. 218.
Formation du traducteur, 1-2, p. 172; 3, p. $313 ; 4$, p. 374 .

Formation professionnelle, 3, p. 330, 332.

Formation terminologique des traducteurs, $1-2$, p. 275.

Formes erronées, 1-2, p. 65 .

Formes graphiques, 1-2, p. 107.

Formes homographes, 1-2, p. 114.

Formes univoques, 1-2, p. 114.

Fournitures de bureau, 4, p. 382

Français d'Amérique du Nord, 1-2, p. 181.

Français vivant, 4, p. 377.

Fréquence, 3, p. 338.

Fréquence aiguë, 4, p. 366.

Fréquence dans le discours, 1-2, p. 126.

Fréquence dans le lexique, 1-2, p. 126.

Fréquence d'emploi, 1-2, p. 131.

Fréquence et étymologie, 1-2, p. 127.

Fréquences (dictionnaire de), 1-2, p. 95.

Fréquences graves, 4, p. 366.

Fresc, 4, p. 360

Frigidaire, 4, p. 360 .

Fuite des cerveaux, 1-2, p. 230.

Fuséologie, 1-2, p. 206.

Gadget, 1-2, p. 93

Galet, 1-2, p. 74

Galon à mesurer, 4 , p. 372.

Garnir, 1-2, p. 78 .

Gavage 1-2, p. 241

Génération morphologique du français, 1-2, p. 287.

Génération syntaxique du français, 1-2, p. 286.

Génie, 1-2, p. 193.

Génie atomique, chimique, etc., 1-2, p. 193.

Génie de l'environnement, 1-2, p. 193.

Gestion, 3, p. 330; 4, p. 390 .

Gestuel, 1-2, p. 207.

Glossaire contextuel, 1-2, p. 237.

Glossaire technique plurilingue, 1-2, p. 261.

Gobelet, 1-2, p. 178.

Gober, 1-2, p. 178 .

Godet, 1-2, p. 178

Gouverneur, 1-2, p. 282.

Grammaire, 1-2, p. 176; 3, p. 311

Grammaire et lexique, 1-2, p. 23.

Grammaire et logique naturelle, 1-2, p. 44.

Grammaire générative, 1-2, p. 202, 207, 216.

Gravillons, 1-2, p. 258.

Grèves, 4 , p. 379.

Gricher, 4, p. 358

Grimper deux étages, 1-2, p. 174

Guerre bactériologique, 1-2, p. 259.

Guichet, 1-2, p. 178.

Guide, 1-2, p. 176.

Haut-parleur, 4, p. 365.

Histoire de la traduction, 4, p. 387.

Historique de la Banque de terminologie, 1-2, p. 271.

Hocher la tête, 1-2, p. 175.

Homographe, 1-2, p. 115.

Homographie, 1-2, p. 117, 119.

Homographie morphologique, 1-2, p. 119.

Homonymie entre deux langues, 1-2, p. 67

Huis clos, 4, p. 380.

Idempotent, 1-2, p. 198.

Identification du texte, 3, p. 309.

Idiolecte, $1-2$, p. 212,232 .

Idiomatique (plan), 3, p. 312. 
Image, 1-2, p. 154.

Image acoustique, 1-2, p. 153.

Image de marque, 4, p. 360.

Image lexicale, 3 , p. 326.

Immobilisation, 1-2. p. 171.

Impartition, 4, p. 379.

Impulsion, 1-2, p. 199.

Incendie, 1-2, p. 191.

Index, 1-2, p. 89, 107

Index alphabétique direct, 1-2, p. 107

Index alphabétique inverse, 1-2, p. 107.

Index bilingues et multilingues, 1-2, p. 94 .

Index chronologique, 1-2, p. 94

Index des fréquences, 1-2, p. 107.

Index des mots, 1-2, p. 94.

Index inverse, 1-2, p. 107.

Index statistique, 1-2, p. 95.

Informatif, 3, p. 309.

Information, 1-2, p. 163.

Information lexicale, 1-2, p. 97 .

Information morphophonémique de l'unité lexicale, 1-2, p. 20.

Information morphosyntactique de l'unité lexicale, $1-2$, p. 20.

Information phonologique de l'unité lexicale $1-2$, p. 20.

Information sémantique de l'unité lexicale, $1-2$, p. 20.

Informations substantives, 1-2, p. 30

Informatique, 1-2, p. 165.

Informatique (application à la lexicologie), $1-2$, p. 87.

Informatique lexicale, 1-2, p. 97.

Intellectuel et affectif, 1-2, p. 172.

Intensité, 3, p. 315, 316.

Intensité augmentative, 3, p. 318

Intensité diminutive, 3, p. 318

Intentionnalité de la langue, 1-2, p. 150.

Intentionnalité signifiante, $1-2$, p. 153 .

Interclasseuse (machine), 1-2, p. 90.

Interpénétration des niveaux de langue, 4, p. 361.

Interpolations, 1-2, p. 68 .

Interprétation et signification, 1-2, p. 69.

Interprétation sémantique, $1-2$, p. 35.

Irrégularité, 1-2, p. 20.

Jeannette, 4 , p. 372 .

Jeu, $1-2$, p. 155 .

Jeu de boules, 4, p. 391.

Joindre, 1-2, p. 171.

Jonc, 4, p. 362 .

Journal interne, 1-2, p. 230.

Journćes internationales de la traduction, 3 , p. 347.

Katz (théorie de), 1-2, p. 146.

Laboratoire chaud, 1-2, p. 241

Lacunes, 1-2, p. 175, 241.

Lacunes dans le réseau cristallographique, 1-2, p. 263.

Langage des machines, 4 , p. 356.

Langage, instrument de communication, 1-2, p. 22.

Langage visuel du rêve, $1-2$, p. 154.

Langue, $1-2$, p. 10

Langue de traduction, 1-2, p. 270.

Langue des affaires, 4 , p. 390.

Langues en contact, 3 , p. 344 .

Langue et parole, 1-2, p. 73.
Langue poétique, 3, p. 322.

Laudatif, 3, p. 319.

Lecteur, 4, p. 366.

Lecteur de caractères, $1-2$, p. 92.

Lecteur multifont, 1-2, p. 109.

Lecteur omnifont, 1-2, p. 109.

Lecteur optique, 1-2, p. 92, 109.

Lecteur phonographique, 4, p. 365, 366.

Lecture à haute voix, 3, p. 313 .

Lecture critique, 3 , p. 313 .

Lecteur de caractères graphiques, 1-2, p. 92.

Lecture optique, 1-2, p. 91, 92, 109.

Lemmatisateur, $1-2$, p. 107.

Lemmatisation, 1-2, p. 93, 107.

Lemmatisation automatique, $1-2$, p. 98.

Lemmatisation des verbes, 1-2, p. 93 .

Lemmatisation et lexique, 1-2, p. 117

Lemmatisation semi-automatique, 1-2, p. 113.

Lemme, 1-2, p. 107.

Lexautom, 1-2, p. 180.

Lexème, 1-2, p. $27,129$.

Lexicalité (définition de), 1-2, p. 13 .

Lexicographie assistée par ordinateur, 1-2, p. 104.

Lexicographie et lexicologie, 1-2, p. 9, 19.

Lexicographie et linguistique, 1-2, p. 139.

Lexicographie historique, 1-2, p. 142.

Lexicographie panchronique, $1-2$, p. 142.

Lexicologie assistée par ordinateur, 1-2, p. 99, 104.

Lexicologie (définition), 1-2, p. 9.

Lexicologie différentielle, $1-2$, p. 171.

Lexicologie et dictionnaires, 1-2, p. 7 .

Lexicologie et informatique, 1-2, p. 87.

Lexicologie et lexicographie, 1-2, p. 9, 19.

Lexicologie et sciences humaines, 1-2, p. 16.

Lexicologie fonctionnelle, 1-2, p. 189.

Lexicologie monolingue et bilingue, 1-2, p. 7.

Lexicologie socioculturelle, 1-2, p. 88 .

Lexicologie (statut), 1-2, p. 9.

Lexicologie structurale, 1-2, p. 237.

Lexicologie (vocation interdisciplinaire de la), 1-2, p. 16.

Lexicométrie, $1-2$, p. 89,95 .

Lexique, 1-2, p. 150, 201, 220, 238; 3, p. 311.

Lexique (analyse du), 1-2, p. 19.

Lexique (articulation du), 1-2, p. 10.

Lexique automatique, 1-2, p. 113, 114.

Lexique automatique (autres fonctions), 1-2, p. 121.

Lexique (définition), 1-2, p. 22.

Lexique (définitions possibles), 1-2, p. 12.

Lexique et glossaire contemporain, 1-2, p. 63 .

Lexique et glossaire historique, 1-2, p. 64 .

Lexique et grammaire, $1-2$, p. 23.

Lexique et productivité, 1-2, p. 217.

Lexique (irrégularité) $1-2$, p. 15.

Lexique (nature du), 1-2, p. 19.

Lexique (régularité), 1-2, p. 15.

Lexique (structures du), 1-2, p. 53.

Lexique (systématicité), 1-2, p. 14

Libération de la sexualité et néologisme, 4, p. 363.

Libre-service, 1-2, p. 230.

Limites typographiques, 1-2, p. 187.

* Linge à presser », 4, p. 372 .

Linguistique, 4 , p. $384,389,391$.

Linguistique de la langue, 1-2, p. 137. 
Linguistique du discours, 1-2, p. 137

Linguistique fonctionnelle, 1-2, p. 151.

Linguistique générative, 1-2, p. 11 .

Linguistique informatique, 1-2, p. 134.

Listage, 1-2, p. 92.

Littérature, 1-2, p. 213

Littérature secondaire, 1-2, p. 67.

Locuteur idéal, 1-2, p. 123.

Logique naturelle, 1-2, p. 35 .

Logique naturelle et grammaire, 1-2, p. 44.

Logolasie, 1-2, p. 230.

Loi de Darbelnet, 1-2, p. 189.

Loisirologie, 1-2, p. 173.

Ludothèque, $1-2$, p. 228

Lunambule, 1-2, p. 228.

Lutte contre les poussières, 1-2, p. 264.

Lycée, 4, p. 369

Machine-outil, 1-2, p. 167

Macrocontexte, 1-2, p. 107; 3, p. 311.

Main, 1-2, p. 269.

Machine à air, 1-2, p. 273.

Manette, 1-2, p. 178.

Manivelle, 1-2, p. 178.

Mannequin, 1-2, p. 266.

Manuscrits de la mer Morte, 1-2, p. 178.

Margelle, 1-2, p. 178 .

Marques d'usage, 1-2, p. 181, 273.

Marques grammaticales, 1-2, p. 273

Marques sémantiques, $1-2$, p. $146,155$.

Masse volumique, 1-2, p. 170 .

Matériaux industriels, 3, p. 340 .

Matériel, 1-2, p. 273.

Mathématiques modernes, 1-2, p. 198; 3, p. 339.

Matrices lexicales, 1-2, p. 215.

Mauvais matin, 1-2, p. 185.

Maximum, 4, p. 380.

Mécanographie et données linguistiques, 1-2, p. 90 .

Médecine physique, 3, p. 340 .

Media, 3, p. 309.

Médiathèque, 1-2, p. 228.

Mélange pauvre, $1-2$, p. 264

Mélioratif et lauditif, 3, p. 318 .

Mémoire, 1-2, p. 92, 168.

Mémoire de masse, 1-2, p. 92, 96.

Mémoire indéfectible, $1-2$, p. 97

Mémoire partielle, 1-2, p. 92 .

Mémoire rapide, 1-2, p. 92.

Mener, 1-2, p. 175.

Mention de date, 1-2, p. 270.

Mention de source. 1-2, p. 270

Mérisme, 1-2, p. 214.

Métadiscours, 1-2, p. 214.

Métalangage, 1-2, p. 214.

Métalangage autonyme, $1-2$, p. 55.

Métalangage de langue, $1-2$, p. 58.

Métalangage du discours, $1-2$, p. 58

Métalangue, 4 , p. 385.

Métaphore, 3, p. 323.

Métaphore éteinte, 3, p. 323.

Métaphorisation, 1-2, p. 210, 219.

Métaux non ferreux, 3 , p. 340 .

Méthodologie de la traduction, 3, p. 314.

Mètre ruban, 4, p. 372.

Microcontexte, 1-2, p. 107; 3, p. 311.

Microfiches, 1-2, p. 92, 96 .

Microphone, 4, p. 365.
Microphone électrodynamique, 1-2, p. 273.

Minéralogie, 3, p. 340.

Mines métalliques, 1-2, p. 264.

Mobilier de bureau, 4, p. 382.

Modalité concrète, $3, \mathrm{p} .325$.

Modèle pour la TA, 1-2, p. 278.

Moderne, 1-2, p. 198

Molette, 4, p. 373

Monosémie, 1-2, p. 239

Morphème, 1-2, p. 13, 122, 207.

Morphème-base, 1-2, p. 206.

Morphème lexical, 1-2, p. 203.

Morphème lexical et sémème, 1-2, p. 214.

Morphologie, 1-2, p. 11.

Mot de base, 1-2, p. 83 .

Mot du discours, 1-2, p. 210.

Mot fantôme, 1-2. p. 65 .

Mot-image, 3, p. 316

Motivation linguistique, 1-2, p. 175.

Mot métalinguistique, 1-2, p. 214.

Mot pivot, 1-2, p. 98 .

Mot (problématique du), 1-2, p. 201.

Mot référentiel, 1-2, p. 209.

Mot sémantique, 1-2, p. 207.

Mots essentiels, 4, p. 377.

Mots grammaticaux, $1-2$, p. 12,13

Mots lexicaux, 1-2, p. 12 .

Mou (physique nucléaire), 1-2, p. 241.

Moyens mécanographiques, 1-2, p. 90.

Multifont, 1-2, p. 109.

Nautique, 4, p. 370

Néologie, 1-2, p. 57, 205, 217, 225.

Néologisme, 1-2, p. 168, 173, 195; 4, p. 355.

Néologisme de consommation, 4, p. 357.

Néologisme de prestige, 4, p. 357.

Néologisme d'ignorance, 4, p. 357.

Néologisme d'image, 4, p. 362

Néologisme d'importation, 1-2, p. 229

Néologisme morphologique, 1-2, p. 249.

Néologisme nécessaire, 4, p. 357.

Néologisme notionnel, 1-2, p. 228.

Néologisme syntagmatique, 1-2, p. 246.

Néologiste, 1-2, p. 233.

Niveaux de langue, 1-2, p. 212; 3, p. 322.

Nivellement par le bas, 4 , p. 357 .

Nom commun, $1-2$, p. 56,58 .

Nomenclature, 1-2, p. 247.

Nomenclature (notion), 1-2, p. 243.

Nom propre, 1-2, p. 54.

Normalisation, 1-2, p. 102, 273, 276.

Normalisation des dictionnaires, 1-2, p. 179.

Normalisation des travaux, 1-2, p. 102.

Norme et dictionnaire, $1-2$, p. 220.

Norme lexicale, $1-2$, p. 214

Notion de terminologie, 1-2, p. 243.

Noyau sếmique, $1-2$, p. 241 .

Nucléaire, 1-2, p. 244.

Nylon, 4, p. 359.

Obsèques, 1-2, p. 172.

Odontologie, 4, p. 390

Ondes électriques, 4, p. 365

Oléoduc, 1-2, p. 258 .

Omnifont, 1-2, p. 109.

Onomasiologie, 1-2, p. 61

Onomatopée, 1-2, p. 57

Opaque, 1-2, p. 241.

Opérande, 1-2, p. 273.

Opérateur, 1-2, p. 43. 
Opposition abstrait-concret, 1-2, p. 264.

Opposition objectivité-subjectivité, 1-2, p. 264.

Ordinateur, 1-2, p. 91.

Organisation, 3 , p. 330

Organisation des cours de traduction, 3, p. 313.

Orignal, 1-2, p. 183

Orme roux, 3, p. 329.

Orthographe, 4, p. 391

Oscillateur d'audiofréquence, 4, p. 366.

Ouvrier/travailleur, 1-2, p. 174.

Padding, 4, p. 372.

Pain francais, 1-2, p. 183.

Paires minimales, 1-2, p. 125.

Papeterie, 1-2, p. 176.

Paradigmes grammaticaux, 1-2, p. 238.

Paradigmes ouverts et fermés, 1-2, p. 12.

Paramètre de ralentissement, 1-2, p. 240.

Parement, 4, p. 373.

Parementure, 4, p. 373

Passage, 1-2, p. 259.

"Passe-carreau ", 4 p. 372.

Pastiches, 1-2, p. 66.

Patate, 1-2, p. 185.

Pathologie dentaire, 3 , p. 339.

Patrons (couture), 4, p. 372

Pattemouille, 4, p. 372.

Péjoratif, 3, p. 319.

Pelage, 1-2, p. 241.

Percussion, 1-2, p. 199.

Père nucléaire, 1-2, p. 241.

Perfectionnement, 3, p. 330, 331.

Péricope, 1-2, p. 112.

Périphérique, 1-2, p. 92.

Perméabilité des catégories grammaticales, 1-2, p. 58.

Perron, 1-2, p. 178.

"Personnel de soutien n, 4, p. 380.

Perte de matière, 1-2, p. 263.

Pesticide, 3, p. 335.

Petit contexte $1-2$, p. 70.

Petite informatique. 1-2, p. 166.

Pétrographie, 3, p. 340.

Phase d'assimilation, 3, p. 309.

Phèmes, $1-2$, p. $1 \Delta 8$.

Philatélie, 3, p. 342.

Phonèmes, 1-2, p. 148.

Phonocanteur, 4, p. 366.

Phonologie, 1-2, p. 19

Photo-combosense, 1-2, p. 100

Phrase, 1-2, p. 12.

Physiologie d'une langue, 4, p. 355.

Physiaue atomique. 1-2, p. 245.

Physique cornusculaire, 1-2, p. 245.

Physique nucléaire, 1-2, p. 245.

Pied-de-roi, 1-2, p. 185

Pierres errantes. 1-2, p. 258.

Pilori, au, 4. p. 374.

Pince à pavier. 4. p. 383.

Piolet, 1-2, p. 178 .

Plages sémantiaues, 3 , p. 311.

*Plan de pension ". 4, p. 379.

Planification, 3 , v. 341.

Pli couché, 4, p. 373 .

Pli rond, 4, p. 373.

Poignée, 1-2, p. 178.

Poison consommable, 1-2, p. 241.

Poison nucléaire, 1-2, p. 241.
Polysémie, 1-2, p. 93, 140, 176, 210, 214.

Pomme de terre, 1-2, p. 185.

Pomme frite, 1-2, p. 185.

Pondération, 1-2, p. 273.

Pondération globale, 1-2, p. 274.

Porter, 1-2, p. 175.

Postillon, 1-2, p. 182

Prédicat converse, 1-2, p. 44.

Préfixes productifs, 1-2, p. 218.

Prendre un virage sur l'aile, 1-2, p. 258.

Présignificatif, 1-2, p. 74 .

"Presser ", 4, p. 372.

Préposé aux écritures, 4, p. 380

Prime d'amplitude, 4, p. 379.

Principe de l'opposition, 1-2, p. 157.

Principes de recherches terminologiques, 1-2, p. 269.

Prix de revient, 4, p. 380.

Problèmes de documentation, 1-2, p. 89.

Problèmes sémantiques, 1-2, p. 69 .

Processus de la traduction, 3 , p. 308

Produits informatiques, $1-2$, p. 166.

"Professionnels », 4, p. 379

Programme, 1-2, p. 93.

Programme d'analyse automatique, 1-2, p. 98.

Programme de contextualisation, 1-2, p. 111.

Programme généralisé, 1-2, p. 103.

Programmerie, 1-2, p. 273.

Promenade, 1-2, p. 177.

Prononciation, 4, p. 360 .

Propre et figuré, 1-2, p. 172.

Prothèse dentaire, 3, p. 339.

Pseudo-dictionnaire de machine, 1-2, p. 113.

Psychanalyse, 4, p. 390.

Psychanalyse et langage, 1-2, p. 154.

Psycho-linguistique, 1-2, p. 212

Pupitre, 1-2, p. 93.

Qualité, 3, p. 315, 319.

Quantificateur, 1-2, p. 39.

Quantificateur universel, 1-2, p. 51.

Québécois, 1-2, p. 182.

Quincaillerie, 1-2, p. 176, 271.

Radio-, 1-2, p. 249.

Rapport de désavantage, 1-2, p. 240.

Rapport d'évocation, 1-2, p. 153.

Rapport prédicatif, 1-2, p. 203.

Rapports expressifs, 3, p. 317.

Rappeler (se), 4, p. 357 .

Rationalisme critique, 1-2, p. 146.

Réacteur à circulation forcée, 1-2, p. 250.

Réacteur à cœur dilué, 1-2, p. 250 .

Réacteur à combustible en suspension, 1-2, p. 250.

Réacteur à eau légère sous pression, 1-2, p. 250.

Réacteur à graphite, 1-2, p. 250.

Réacteur atomique ou nucléaire, 1-2, p. 245.

Réacteur à tubes de force, 1-2, p. 250 .

Réadaptation, 3, p. 340.

Réalités extralinguistiques, 1-2, p. 149.

Réarrangement en TA, 1-2, p. 284.

Rebord de fenêtre, 1-2, p. 177.

Recherche documentaire, 1-2, p. 104

Recherche lexicologique, 1-2, p. 103.

Reconstructions fausses, 1-2, p. 65 .

Recopiage, 1-2, p. 109.

Récréation, 1-2, p. 173.

Récréologie, 1-2, p. 173. 
Rééducation, 3, p. 340.

Référence bibliographique, 1-2, p. 270.

Référence dénominative, 1-2, p. 22.

Référent, 1-2, p. 214.

Réfrigérateur, 4 , p. 360.

Registre, 1-2, p. 92.

Regroupement notionnel, 1-2, p. 98.

Regroupement thématique, 1-2, p. 98 .

Reichling (théorie de), 1-2, p. 141.

Relâchement des rapports étymologiques, 1-2, p. 244.

Relation, 3, p. 320.

Relation de filiation, 1-2, p. 207.

Relation géographique, 3, p. 315,321 .

Relation linguistique, 3 , p. 316,322 .

Relation métalinguistique, 3 , p. 320 .

Relation sociale, 3, p. 315, 321 .

Relations professionnelles, 4 , p. 378.

Relations syntaxiques, $1-2$, p. 42

Relation temporelle, 3, p. 315, 321 .

Relation traditionnelle, 3, p. 315,322 .

Remue-méninges, $1-2$, p. 230

Renaissance de mots archaïques, 4, p. 359.

Rendement fonctionnel, $1-2$, p. 125.

Repasser à la pattemouille, 4, p. 372

Répertoires partiels, $1-2$, p. 100

Reporteuse, 1-2, p. 90, 91 .

Représentation verbale, 1-2, p. 153.

Réprimander, 1-2, p. 173

Reproduction, 1-2, p. 177.

Reproductrice, $1-2$, p. 90.

Reproductrice (machine), 1-2, p. 91.

Reprographie, 1-2, p. 177.

Reprographier, 1-2, p. 236

Reprographiste, 1-2, p. 236 .

Restitution, 3, p. 312 .

Résultats du dépouillement, 1-2, p. 108.

Retouches, 4, p. 372.

Revers, 4, p. 373.

Ricrac, 4, p. 372.

Rôle des citations, $1-2$, p. 75 .

Roulette, 4, p. 373.

Rythme, 3, p. 313

Salaire, 4, p. 380.

Salarié, 4, p. 379

Saucisson, 4 , p. 358

Saut de lit, 1-2, p. 176 .

Sauvetage, 4, p. 371 .

Sauvetage nautique, 4 , p. 371 .

Science-fictionnesaue, 1-2, p. 227.

Scripteur, 1-2 p. 174

Secondaire V, 4, p. 370

Sécurité nautique, 4, p. 371.

Sélectivité, 3, p. 336.

Sémantique, $1-2$, p. 9

Sémantique, (n.m.), 1-2, p. 208.

Sémantique et logique naturelle, 1-2, p. 35

Sémantique lexicale, $1-2$, p. 156.

Sémantique structurale, 1-2, p. 151.

Sémasiologie, 1-2, p. 61 .

Sème, 1-2, p. 147.

S̀̀me connotatif, 1-2, p. 157.

Sème d'appel, 1-2, p. 157.

Sème dénotatif, 1-2, p. 157.

Sème d'expression, 1-2, p. 157.

Sémème, 12, p. 148, 156, 207, 208, 220.

Sentence, 1-2, p. 12.

Série lexicale transformationnelle, 1-2, p. 205.
Série lexicologique, 1-2, p. 180.

Série métalinguistique, 1-2, p. 59.

Série sémantique, 1-2, p. 180.

¿ Set de chambre », 1-2, p. 175.

Sexplosion, 1-2, p. 228.

Sifflement, 1-2, p. 177

Signal d'audiofréquence, 4 , p. 365 .

Signal électrique, 4 , p. 365 .

Signal électroacoustique, 4 , p. 365.

Signature des fiches, 1-2, p. 274.

Signe, 4, p. 385.

Signe lexical, 1-2, p. 9

Signème, $1-2$, p. 61 .

Signifiant, signifié, référent, 1-2, p. 124.

Signification, 1-2, p. 69, 239.

Signification (classement), 1-2, p. 76.

Signification (définition), 1-2, p. 140 .

Signification des échantillons, 1-2, p. 136

Signification du mot, 1-2, p. 139.

Signification et désignation, 1-2, p. 150

Signifié du nom propre, 1-2, p. 58 .

Signifié lexical, 1-2, p. 17.

Simplicité, 1-2, p. 195.

"Ski aquatique », 4, p. 371

Ski nautique, 4 , p. 371 .

Société des traducteurs du Québec (S.T.Q.), 3, p. 348.

Sociologie, 4, p. 390.

Soldat/combattant, 1-2, p. 174

Sortie de bal, 1-2, p. 176.

Sortie des données, 1-2, p. 92.

Soupirail, 1-2, p. 178

Sources, 4 , p. 383 .

Sous-motivation du français, 1-2, p. 176.

Sous-sémème, $1-2$, p. 220

Soutenir, 1-2, p. 173.

Soutien-gorge pigeonnant, 1-2, p. 259.

Spectre audible, 4 , p. 366

Sports nautiques, 4 , p. 371

Stagflation, 1-2, p. 228.

Statistique lexicale, $1-2$, p. $89,95$.

Statistique lexicographique, 1-2, p. 127.

Statut de la lexicologie, 1-2, p. 9 .

Statut des dérivés, $1-2$, p. 82 .

Sténographie, 3, p. 340 .

Stratigraphie, 3 , p. 340.

Structuralisme asémantique, $1-2$, p. 35 .

Structuration de la substance du contenu, 1-2, p. 10.

Structuration sémantique du lexique, 1-2, p. 7 , 145.

Structure analogique, 1-2, p. 271.

Structure associative, 1-2, p. 271 .

Structure cognitive, $1-2$, p. 14.

Structure de sèmes, $1-2$, p. 156.

Structure du lexique, 1-2, p. 53.

Structure lexicale, 1-2, p. 237.

Structure lexicale des terminologies, 1-2, p. 249.

Structure linguistique, 1-2, p. 14.

Stylet pointeur, 1-2, p. 93 .

Stylo dosimètre, 1-2, p. 249.

Stylographe à bille, 4 , p. 360 .

Substance du contenu, 1-2, p. 10.

Substantif, 1-2, p. 54 .

Substitution des mots d'emprunt, 4, p. 358.

Surface, 1-2, p. 174

Suffixation, 1-2, p. 248. 
Suite, 1-2, p. 230

Superlatif, 1-2, p. 197

Supporter, 1-2, p. 173

Surcaractérisation, 1-2, p. 176.

Surchauffe, 1-2, p. 248.

Synapsie, 1-2, p. 249.

Synchronie, 1-2, p. 10

Synonyme, 1-2, p. 209.

Synonymie, 1-2, p. 209.

Synonymie et complémentarité, 1-2, p. 71.

Syntagmes épithétiques, 1-2, p. 249.

Syntaxe, $1-2$, p. 20

Syntaxe de profondeur, 1-2, p. 40

Syntaxe et lexique, $1-2$, p. 145 .

Syntaxique (n. m.), 1-2, p. 208.

Système lexical, 1-2, p. 32 .

Table de concordance, 1-2, p. 94 .

Tabulatrice, 1-2, p. 90,91 .

Tagmème, 1-2, p. 23

Tambour, 1-2, p. 92

Tasse, $1-2$, p. 178 .

TAUM, 1-2, p. 277

Taxi, 4 , p. 360

Température ambiante, 1-2, p. 263.

Tendance à l'abstraction, 1-2, p. 265.

Terme, 1-2, p. 27.

Termes de bureau, 4, p. 381.

Terminal à clavier, 1-2, p. 93.

Terminal CTR, 1-2, p. 109.

Terminal vidéo, $1-2$, p. 92, 112.

Terminologie, 1-2, p. 232, 269.

Terminologie et nomenclature, 1-2, p. 242.

Terminologie et traduction, 1-2, p. 271.

Terminologie (notion), 1-2, p. 243.

Terminologie technique, 1-2, p. 237.

Terminologie scientifique et langue commune, $1-2$, p. 240 .

Tête de lecture, 4 , p. 366 .

Texte, 1-2, p. 67 .

Texte exhortatif, 3 , p. 309 .

Texte expressif, 3, p. 309

Texte général, 3, p. 314

Texte informatif, 3 , p. 309.

Texte original, $1-2$, p. 270.

Texte référentiel, 3, p. 309

Texte religieux, 3, p. 309.

Texte spécialisé, 3 , p. 314.

Théorie de Katz, 1-2, p. 146

Théorie psychologique de Freud, 1-2, p. 154

Thésaurus, 1-2, p. 128.

Tine, 1-2, p. 177.

Tissu urbain, 1-2, p. 197.

Tractation, 4, p. 379.

Traducteur, 4, p. 365.

Traduction automatique (TA), 1-2, p. 129, 277.

Traduction humaine, 1-2, p. 161.

Traductrice (mécanographie), 1-2, p. 90.

Traduire, 4, p. 365.

Traîneau, 1-2, p. 175 .

Traitement linguistique en TA, 1-2, p. 278.

Trame, 4, p. 373

Transducteur, 4, p. 365

Transformation, 1-2, p. 52 .

Transformationalisme asémantique, 1-2, p. 35 .
Transformationaliste, 1-2, p. 20.

Transformer, 4, p. 365 .

Transformer les ondes sonores, 1-2, p. 269.

Transparent, 1-2, p. 241.

Trapèze de Heger, 1-2, p. 61.

Travail du terminologue, 1-2, p. 168

Travailleur, 1-2, p. 174

Travailleur à toutes mains qualifié, 1-2, p. 174.

Travailleur déclassé, 4, p. 379

Travailleurs sociaux, 1-2, p. 174 .

Travailleuse familiale, 1-2, p. 174.

Trésor de la langue française, 1-2, p. 97, 136.

Tri, 1-2, p. 91.

Triangle d'Ullmann, 1-2, p. 140.

Tri de fichier, $1-2$, p. 94 .

Tri des homographes, 1-2, p. 126.

Trieuse, $1-2$, p. 90

Trombone, 4, p. 383.

Trous en formation, 1-2, p. 258.

Tube à essai, $1-2$, p. 270

Turbulent, 1-2, p. 173.

Type de discours, 1-2, p. 212

Unité analytique, 1-2, p. 25

Unité analytique du lexique, 1-2, p. 27.

Unité d'affichage, 1-2, p. 92 .

Unité d'affichage à stylet pointeur, 1-2, p. 93.

Unité comportementielle, 1-2, p. 24.

Unité de base du dictionnaire génétique, 1-2, p. 63.

Unité de classe fermée, 1-2, p. 53.

Unité de classe ouverte, 1-2, p. 53 .

*Unité de négociation ", 4, p. 379

Unité dénominative, 1-2, p. 25.

Unité de traduction, 3, p. 311.

Unité de traitement, 1-2, p. 93.

Unité fonctionnelle du discours, 1-2, p. 11.

Unité lexicale (différenciation), 1-2, p. 24.

Unité lexicale et information, 1-2, p. 20.

Unité lexicale (propriétés syntaxiques), 1-2, p. 47.

Unité-signe, 1-2, p. 10.

Univers, 1-2, p. 197

Universalité, 3 , p. 336

Univoque, 1-2, p. 115.

Urf, 4, p. 356.

Usages régionaux, 1-2, p. 182.

Usance, 1-2, p. 230

Vagir, 1-2, p. 178.

Valence lexicale, 3, p. 338.

Valeur, 1-2, p. 239.

Variable grammaticale, 1-2, p. 279

Variantes graphiques, 1-2, p. 119

Variable référentielle, 1-2, p. 45.

Ventouse, 1-2, p. 178

Véronal, 4 , p. 359

Version anglaise, 4, p. 390

Vidéo, 1-2, p. 92

Vie amoureuse, 4 , p. 390.

Viscéral, 1-2, p. 173 .

Vocabulaire général d'orientation scientifique (V.G.O.S.), 1-2, p. 213, 269.

Volapuk, 4 , p. 356.

Vulgarisation, 1-2, p. 192.

Yachting, 4, p. 371 .

Zip, 4, p. 372 . 